
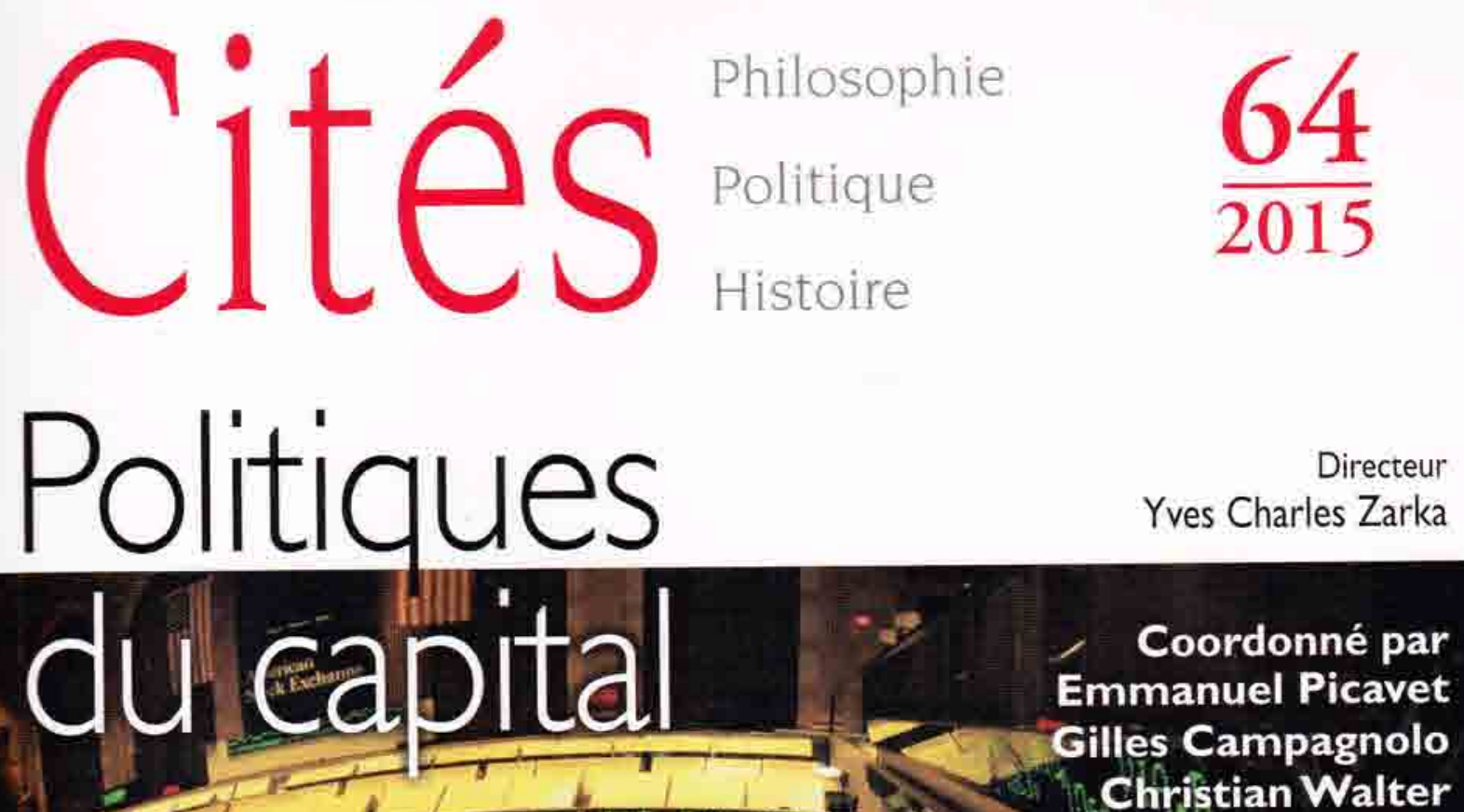

Coordonné par Emmanuel Picavet Gilles Campagnolo Christian Walter

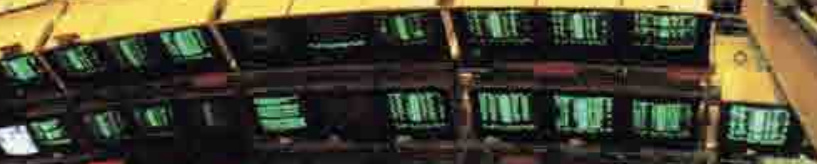

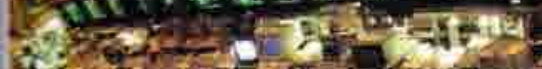

Réflexions sur Le Capital au xxie siècle Réponse de

Yves Charles Zarka Un capital sans capitalisme? 


\title{
La normalisation comptable internationale : de l'État de droit au droit sans l'État ${ }^{1}$
}

\author{
Yvonne Muller-Lagarde
}

Longtemps demeurée confidentielle et réservée à quelques experts, la normalisation comptable internationale est devenue objet du discours politique à la suite de l'adoption par la Commission européenne, en 2002, du référentiel comptable international produit par un organisme privé ; elle alimentera, à la suite de la crise financière de 2008 , une doctrine hostile accusant les normes comptables internationales d'avoir amplifié la crise et accéléré sa propagation ${ }^{2}$. La promotion de ces normes, soudainement portées au cour des débats politiques internationaux, notamment ceux du G20, a permis de dépasser leur caractère technique pour s'interroger sur les croyances et valeurs qu'elles véhiculent au nom d'un certain ordre juste ${ }^{3}$. Hissées par certains au rang de "bien collectif" " ou de "bien commun" ", définies par d'autres comme " objet régalien ${ }^{6}$ " les normes comptables sont

1. L. Cohen-Tanugi, Le Droit sans l'État, Paris, Puf, 2007.

2. D. Marteau et P. Morand, Normes comptables et crise financière, Propositions pour une réforme du système de régulation comptable, Rapport au Ministre de l'économie, de l'Industrie et de l'Emploi, 13 octobre 2009 , p. 20.

3. Lexpression est empruntée à A. Supiot, in Homo juridicus. Essai sur la fanction anthropologique du droit, Paris, Seuil, 2005, p. 265.

4. C. Hoarau er R. Teller, IFRS : «Les normes comptables du nouvel ordre économique global ", Comptabilité, Contróle, Audit, 2007/3, p. 13 sq.

5. A. Burlaud et B. Colasse, Réponse aux commentaires sur " Normalisation comptable internationale : le retour du politique? ", Comptabilité, Contróle, Audit, tome XVII, déc. 2011, p. 115 sq., spéc. p.122.

6. E. Chiapello et K. Medjad, "Une privatisation inédite de la norme : le cas de la politique comptable européenne ", Sociologie du travail, vol. 49, janvier-mars 2007, p. 46. 
désormais présentées, à travers l'information qu'elles véhiculent, comme participant d'une compétition intellectuelle mondiale visant à influencer, à travers des choix stratégiques, le fonctionnement de l'économie mondiale.

Peu investie par la doctrine juridique, la critique de la normalisation comptable internationale nourrit pourtant les réflexions, dans une économie globalisée, sur la mutation du droit qui s'inscrit, de façon plus globale, dans une mutation profonde des sociétés occidentales entrées depuis la deuxième moitié $\mathrm{du} \mathrm{xx}^{c}$ siècle et sous l'effet de la mondialisation, dans l'ère de ce qu'il est convenu d'appeler, le postmodernisme. Ce mouvement, théorisé notamment dans l'ouvrage de Jean-François Lyotard La Condition post-moderne (1979), annoncerait la sortie du monde moderne qui a placé, depuis les traités de Westphalie du Xvir siècle, l'État au centre du système politique et juridique. Caractérisée par l'émergence d'une nouvelle forme d'organisation politique globale, l'ère post-moderne emporte une remise en cause de la conception traditionnelle de l'État et bouleverse sa relation au droit, modelée dans le concept d'État de droit. Celui-ci postule un État construit par le droit qu'il édicte mais aussi qui le limite. Il renvoie à un ordre juridique hiérarchisé, expression de la souveraineté étatique, tel que systématisé par Hans Kelsen"

Désormais, la société post-moderne s'inscrit dans un espace géographique diffus et dominé par le pluralisme juridique tel que l'a exposé Santi Romano"; parce que l'État est relayé, supplanté ou encore concurrencé dans la production du droit ${ }^{10}$, celui-ci ne procède plus exclusivement de la souveraineté législative de la nation ${ }^{11}$ révélant un éclatement de la notion d'intérêt général. Replacer le processus de normalisation comptable internationale entre ce double phénomène de pluralisme juridique ${ }^{12}$ et d'éclatement de la notion d'intérêt général peut, nous semble-t-il, contribuer à

7. J-F. Lepetit, « Proposition de réforme du dispositif français de normalisation comptable $"$, 6 avril 2007, p. 1-4.

8. H. Kelsen, Théorie pure du droit (1934), trad. de la $2{ }^{\circ}$ éd., 1962.

9. LOrdre juridique, Paris, Dalloz, 1975.

10. J. Chevallier, L'État post-moderne, Paris, LGDJ, 4' éd., 2014, p. 124.

11. A. Supiot, Homo juridicus, op. cit., p. 27.

12. S. Kott, "Le concept de système juridico-comprable à l'épreuve de la normalisation internationale ", in Y. Muller-Lagarde (dir.), Normalisation comptable, Actualités et enjeux, Thomson Reuters, 2014, p. 39. 
faire émerger une nouvelle évolution en lien avec la responsabilité sociale des entreprises.

\section{NORMALISATION COMPTABLE INTERNATIONALE ET PLURALISME JURIDIQUE}

Les normes comptables internationales (IFRS pour International Financial Reporting Standards) ont pour objectif de permettre, à travers le monde, la compatibilité et la comparabilité de l'information financière diffusée par les grandes entreprises sur les marchés financiers. Elles sont appliquées, à ce jour, dans 138 pays dont les États membres de l'Union européenne, représentant plus de $97 \%$ du produit intérieur brut mondial. Si elles ont vocation à s'appliquer, en priorité, aux comptes consolidés des entreprises transnationales qui agissent sur les marchés internationaux, des processus de convergence avec les comptes individuels des sociétés et les comptes publics, relevant naturellement des législations nationales, ont été lancés marquant une stratégie d'expansion sans précédent.

$\mathrm{La}$ normalisation d'un référentiel comptable à vocation mondiale et marqué d'une grande technicité, ne pouvait être le produit d'un seul État incapable de dépasser l'obstacle des souverainetés étatiques et de leurs intérêts divergents. Aussi, dès les années 1960, émerge, à travers les congrès internationaux et les publications professionnelles et académiques, une recherche de normes comptables capables de fournir un langage commun dans le champ de l'économie mondiale. L'harmonisation comptable tentée par la Commission européenne dans les années 1970, et initiée dans le cadre plus large d'une harmonisation du droit de sociérés va échouer, celle-ci étant incapable de dépasser les divergences des États-membres et, précisément, l'opposition entre le système juridique anglo-saxon et le système juridique continental ${ }^{13}$. L'échec européen va laisser place aux initiatives particulièrement dynamiques de la profession comptable. Parce que dans les pays de droit continental comme la France, la comptabilité est marquée par l'emprise étatique, à travers notamment les premières planifications, l'initiative viendra d'Angleterre où la culture juridique, dominée par la Common Law, permet de penser la loi sans l'État. Précisément, dès la révolution industrielle, le gouvernement laisse 
à l'entreprise le soin de déterminer l'information financière à fournir aux actionnaires. Se développe alors un réseau entre les associations professionnelles comptables qui proposent de fournir des normes de qualité, orientées vers les besoins des grandes sociétés ${ }^{14}$. Dans ce contexte, Henry Benson, associé du Cabinet Coopers and Lybrand, fonde en 1973 avec les associations professionnelles de plusieurs pays, un organisme privé, l'International Accounting Standard Committee (IASC), devenu en 2001 l'International Accounting Standard Board (IASB), aux fins de répondre, sous la pression de l'économie globale, à la demande de normes internationales. C'est à ce moment que se dessinent les grandes lignes de la comptabilité qui vont marquer la normalisation mondiale.

Très rapidement, prenant acte de son échec dans l'harmonisation comptable, la Commission européenne déclare dans une communication (2000) confier celle-ci à l'IASC. Adoptées par la Commission dans un règlement de $2002^{15}$, les normes de l'IASC deviennent obligatoires à toutes les sociétés cotées européennes à partir de 2005 entraînant une vague d'adhésions similaires dans le monde, notamment par les pays développés.

Dans le champ ouvert de la mondialisation, l'action de l'IASB va s'exercer, en marge des États, mais conjointement avec d'autres acteurs politiques ou privés comme l'Organisation des Nations unies (ONU) ou l'Organisation de coopération et de développement économique (OCDE). Le processus de normalisation comptable internationale est donc moins le résultat d'une stratégie d'éviction des États par le normalisateur privé, que l'expression d'une transformation du droit, marquée par une complémentarité complexe des différents acteurs et modes de régulation; il participe, en cela, d'un mouvement plus global de régulation transnationale privée ${ }^{16}$, illustrant le passage d'un État régulateur au capitalisme régulateur nullement spécifique à la comptabilité comme le montre la régulation d'internet, de l'environnement, de la sécurité alimentaire ou encore de la propriété industrielle. Mais ce déplacement des sources du droit vers des pouvoirs privés économiques replace la question de la souveraineté au cour de la régulation par le droit. Partagée, fragmentée, la souveraineté est remise en cause et pose inévitablement la question de

14. W. Peter, "Le rôle de linformation financière dans le monde anglo-saxon ", in $L a$ Comptabilité anglo-saxonne, Paris, La Découverte, 2008.

15. Règlement (CE) n ${ }^{\circ} 1606 / 2002$ du 16 juillet 2002 .

16. C. Fabrizio, - Les nouveaux fondements de la régulation transnationale privée ", in Revue internationale de droit économique 1/2013 (t. XXVII), p. 129 sq. 
la légitimité du processus de régulation et, au-delà, de la détermination de l'intérêt général qui la fonde.

\section{NORMALISATION COMPTABLE INTERNATIONALE} ET LA RECHERCHE DE L'INTÉRÊT GÊNÉRAL

Outre le pluralisme juridique, la mondialisation se caractérise également par une financiarisation de l'économie et, plus spécialement du processus d'accumulation du capital qui aurait conduit à la "grande déformation " de l'entreprise transnationale ${ }^{17}$; le développement des marchés financiers a, en effet, rendu l'accès au capital plus rapide et plus simple, via de multiples instruments financiers plus ou moins sophistiqués. Partant, le régime de croissance de l'entreprise est désormais fondé moins sur les investissements productifs que sur les investissements financiers. Cette révolution financière a conduit au nouveau paradigme de la valeur financière que les dirigeants doivent maximiser, au détriment de la valeur productive. Cette position, formalisée par la théorie de l'agence développée par Jensen et Meckling dans les années 1970, a conduit à privilégier, à travers le mouvement de corporate governance, un mode de gouvernance de l'entreprise qui privilégie la relation de l'actionnaire et du manager au détriment de celle du manager et du salarié ${ }^{18}$. L'idéologie financière portée par l'économie mondialisée marque la domination du capitalisme anglo-américain, tel qu'il émerge dans les années 1980, par opposition au capitalisme rhénan, pour reprendre la distinction proposée par Michel Albert. Ce capitalisme donne en effet primauté aux marchés financiers, c'est-à-dire aux investisseurs qui reprennent le contrôle de la gouvernance des entreprises ${ }^{19}$. Or parce qu'elle est issue d'organisations professionnelles de culture anglo-saxonne et vise à satisfaire les besoins d'informations financières des grandes sociétés sur les marchés mondiaux, la normalisation comptable internationale est clairement orientée vers le capitalisme financier comme en témoigne la politique comptable exposée, par l'IASC, dans son cadre conceptuel dès 1989. Que dit-il ? Globalement que l'information financière vise à fournir, en priorité, la

17. "L'émergence de l'entreprise-marchandise ", Revue de droit du travail, 2010, p. 22.

18. Entretien avec $O$. Favereau et $A$. Hatchuel, " Management et financiarisation. Des entrerises "déformées" ", Cadres-Cfdt, n ${ }^{\circ}$ 445-446, septembre 2011, p. 11.

19. B. Colasse, "La vision de l'entreprise et de sa gouvernance comme enjeu de la normaliation comptable internationale s, Conventions, IHEJ, octobre 2012. 
meilleure information aux investisseurs afin de les aider à prendre des décisions économiques mais aussi de juger la gestion des dirigeants. Autrement dit, la comptabilité est destinée à fournir une évaluation de la situation financière et de la performance de l'entreprise. Centrée sur la valeur de l'entreprise, cette approche s'oppose à l'approche continentale plus juridique et patrimoniale et, surtout partenariale car destinée à rendre des comptes à une diversité d'utilisateurs. Le choix du modèle anglo-saxon détermine ainsi la représentation de l'entreprise présentée comme un actif qu'il convient de valoriser et non pas une institution appelée à prendre des décisions économiques. Cette philosophie comptable conduit à l'adoption de méthodes comptables sur lesquelles se sont cristallisées, notamment après la crise de 2008, les critiques. Il s'agit principalement de l'évaluation des actifs et passifs à la juste valeur (fair value) ; celle-ci est fondée soit sur les prix des marchés, soit sur des valeurs modélisées en l'absence de marché actif. La juste valeur doit permettre d'obtenir des valeurs comprables proches de la réalité économique et traduire la richesse propre de l'entreprise. Elle est préférée à la méthode traditionnelle (que le cadre conceptuel n'écarte pas) d'évaluation au coût historique qui consiste à enregistrer des éléments à la valeur d'origine.

Très vite, les normes internationales sont accusées de véhiculer une vision actionnariale de l'entreprise et d'être les « instruments du capitalisme financier ${ }^{20}$ ". Dans ces conditions, la décision de la Commission européenne de déléguer la normalisation comptable à un simple organisme privé devient une affaire politique. En 2003, Jacques Chirac, président de la République, écrit à Romano Prodi, président de la Commission européenne, pour lui demander de ne pas approuver les normes internationales sur les instruments financiers et de revoir le rôle de la Commission dans le processus de normalisation. La crise de 2008 va raviver les critiques qui dénoncent l'abandon par l'Union européenne de sa souveraineté. Celle-ci va réagir dès 2013, avec la volonté de renforcer le rôle de l'Union européenne dans le processus de normalisation comptable ${ }^{21}$.

C'est admettre que la normalisation comptable internationale, appuyée sur une régulation post-moderne détachée de la souveraineté étatique, souffre d'un déficit de légitimité. S'il est difficile de la puiser dans la mise en place d'un processus démocratique, elle pourrait en revanche venir d'un

20. M. Capron (dir.), Les Normes comptables internationales, instruments du capitalisme financier, Paris, La Découverte, 2005.

21. Maystadt Report, Should IFRS standards be more "European "?, octobre 2013. 
autre acteur de la régulation mondiale, l'entreprise transnationale ellemême avec le mouvement de la responsabilité sociale de l'entreprise.

\section{I.A RECHERCHE D'UNE LÊGITIMITÉ PROCÉDURALE}

Dans la régulation post-moderne, le droit tend à puiser sa légitimité dans la procédure par laquelle il est produit, en sollicitant le dialogue avec les destinataires de la norme. La légitimité procédurale pourrait alors annoncer l'ère des "démocraties dialogiques ${ }^{22}$ ". Cela explique sans doute que tout au long de son histoire, l'IASC va procéder, parfois sous la pression de la commission européenne, à des modifications de sa gouvernance aux fins d'associer davantage les parties prenantes. Le normalisateur n'hésite pas alors à emprunter aux catégories juridiques de l'État " pour asseoir symboliquement sa légitimité politique ${ }^{23}$ ". L'une des modifications les plus importantes est la transformation, en 2001, de l'IASC en fondation, la fondation IFRS dotée d'une constitution plusieurs fois révisée depuis. Sa gouvernance, détachée ainsi de la profession comptable, est assurée par des Trustees qui veillent à l'application de la constitution, tandis que l'élaboration des normes est confiée au bureau (IASB). Suivra la création de nouveaux organes de supervision et de consultation. De même et à l'instar du modèle de gouvernance de l'Union européenne mais aussi de nombreuses organisations non gouvernementales, I'IASB a développé au fil des années une procédure standardisée d'élaboration des normes (due process) qui comprend des phases de discussion et de consultation, ainsi qu'une évaluation postérieure de la norme. Parallèlement, l'intérêt des politiques européens pour la normalisation comptable va s'affirmer franchement à partir de 2006 et conduire à associer les parlementaires à la procédure d'adoption des normes comptables. En ce sens, l'Union européenne soumet sa contribution financière à la fondation IFRS ${ }^{24}$ à un contrôle des objectifs poursuivis par celle-ci et de tout changement qui serait introduit dans le cadre conceptuel ${ }^{25}$.

22. G. Timsit, "La régulation. La notion et le phénomène ", Revue française d'administration publique, 2004, p. 5 sq.

23. L. Zevounou, « La normalisation comptable internationale se fait-elle réellement en marge du droit ", in Y. Muller-Lagarde (dir.), Normalisation comptables. Actualités et enjeux, op. cit., p. 23 sq.

24. Règlement (UE) préc., $\$ 17$ et $\$ 24$.

25. Y. Muller-Lagarde, " Les enjeux de la révision du cadre conceptuel du normalisateur comptable international ", Droit des sociétés, 2015, p. 6. 
Pour autant, les efforts vers une « démocratie procédurale " n'épuisent pas le débat de la légitimité de la normalisation comptable internationale dès lors que les principes fondateurs des normes, qui semblent converger, n'ont pas semble-t-il, vocation à être discutés. Ainsi la constitution de la fondation IFRS comme le projet de cadre conceptuel réaffirment la primauté des investisseurs qui demeurent les destinataires privilégiés. De son côté, dans une communication sur sa stratégie en matière d'information financière (2000), la Commission européenne, après avoir envisagé "de laisser aux forces du marché " le soin de déterminer quel est, entre les normes de l'IASB et les normes comptables américaines, le corps de normes d'information financière plus adapté, y renonçait, non pour des raisons politiques mais " pour éviter de prolonger inutilement la durée de compétition entre les deux corps de normes ". Dans le même document, elle affirmait que les "normes comptables doivent satisfaire aux besoins des investisseurs et être compatibles avec les évolutions au niveau international " $(\$ 13)$. Si, dans son règlement de 2002 (préc.), elle mentionne la soumission desdites normes à l'intérêt public européen, la commission affirme cependant " qu'elles doivent satisfaire aux critères $[. .$.$] exigés de$ l'information financière nécessaire à la prise de décisions économiques et à l'évaluation de la gestion des dirigeants de société ". Dès lors, si d'aucuns appellent un " retour au politique " dans la normalisation comptable internationale afin de replacer l'intérêt général ${ }^{26}$ au cour du processus ${ }^{27}$, encore faut-il définir l'intérêt général ici visé.

\section{À LA RECHERCHE DE L'INTÉRÊT GÉNÉRAL}

La notion d'intérêt général doit être appréciée à la lumière des mutations de l'État et de sa capacité, désormais contestée, à faire prévaloir un tel intérêt. Anticipant les débats sur la normalisation comptable, les juristes s'interrogent dès la fin du XX siècle : " Pourquoi l'État aurait-il le monopole de l'intérêt général ?28 " acceptant l'émergence d'acteurs nouveaux dans la

26. L. El Mehdi, « Comptabilité politique ou le droit comptable au service de l'intérêt général ", in Vie \& Sciences de l'entreprise, 3/2013, p. 77 sq.

27. ANC, "Entretiens avec J. Haas ", in Confrontations Europe, $n^{\circ} 97$, p. 1.

28. Conseil d'État, Rapport public 1999, "Lintérêt général ", La Documentation française, 1999, p. 237 sq, spéc., p. 316. 
formulation de cet intérêt. Aussi ${ }^{29}$, un ancien membre de l'IASB, pouvait affirmer en 2012 " qu'il n'y a pas de privilège immanent de l'État sur l'intérêt général $[\ldots]$ ", et d'ajouter "qu'un organisme non étatique, pourvu qu'il soit bien gouverné, transparent et puisse résister aux lobbies peut travailler dans l'intérêt général [...] \%. C'est ici faire prévaloir la conception utilitariste de l'intérêt général sur la conception volontariste.

L'émergence d'un État post-moderne, banalisé et concurrencé par d'autres pouvoirs fragilise, en effet, la conception volontariste de l'intérêt général caractéristique du modèle juridique continental et notamment français. Cette conception place l'État au cœur de la notion - juridique et politique - d'intérêt général ; expression de la volonté générale au sens ousseauiste, l'intérêt général, déterminé et garanti par l'État résulte du tépassement des intérêts particuliers tels qu'ils s'expriment sur le marché. ?arce qu' elle est plus souple et mieux à même de s'adapter à la globalisation conomique ${ }^{30}$, prédomine désormais la conception utilitariste er libérale de 'intérêt général, caractéristique du modèle juridique anglo-saxon. Pensée ur le modèle de l'activité économique, cette conception n'accorde qu'un ôle subsidiaire à l'État dans la définition de l'intérêt général qui n'est que la omme des intérêts particuliers. La confrontation, sur le marché, des intéêts des agents économiques suffit à dégager la notion. C'est naturellement : modèle anglo-saxon de l'intérêt général, dans lequel l'État est en retrait, ui domine l'actuel système de normalisation comptable. L'éclatement des ouvoirs entre différentes autorités dont les intérêts se superposent, exlique l'absence d'une notion unique et supérieure d'un intérêt général qui résiderait à la fabrication de la norme comptable. Le retour au politique ans la normalisation comptable, souhaité par de nombreux acteurs, vise ès clairement à remettre en cause la primauté donné à l'intérêt du marché ir une prise en compte des intérêts des parties prenantes.

Il reste que la poursuite d'un objectif d'intérêt général ou d'intérêt ublic continue d'être affirmée par chacun des acteurs du processus de rmalisation comptable internationale sans qu'il soit possible de dégager 1e notion consensuelle, que l'État n'est plus en capacité de donner ici. nsi, la constitution de la fondation IFRS ${ }^{31}$ affirme que le développement ondial d'un jeu unique de normes doit se faire "dans l'intérêt public "

29. Gilbert Gélard " La légitimité des IFRS : L'intérêt général, concepts et "due pracess" ", in vuller-Lagarde (dir.), Normalisation comptable, Actualités et enjeux, op, cit., p. 108.

30. J. Chevallier, L'Etat post-moderne, op. cit., p. 137.

31. Constitution de l'IFRS Foundation disponible sur www.focusIFRS.com. La constitution a révisée sept fois, la dernière révision datant de 2013. 
("public interest ") sans pour autant que cet intérêt soit défini de manière spécifique. Dans une conférence récente (2015), le président de l'IASB s'est toutefois attaché à le préciser, soulignant que leur travail sert l'intérêt public " en favorisant la confiance, la croissance et la stabilité financière à long terme dans l'économie globale ". L'intérêt public de la fondation IFRS est donc de contribuer à la stabilité de l'économie mondiale " au-delà de la poursuite d'idéologies comptables ou d'intérêts nationaux. "Notons que le Parlement et le Conseil de l'Union européenne font eux-mêmes référence, dans un règlement de $2014^{32}$, à la " mission d'intérêt public " de l'IFRS Foundation validant, si besoin était, son rôle politique.

De son côté, le règlement européen de 2002 (préc.) soumet l'adoption des normes comptables au respect de "l'intérêt public européen ${ }^{33}$ " sans donner de définition de l'intérêt public visé et la notion reste, semble-t-il, à "inventer ${ }^{34}$ ". Il ressort toutefois des nombreuses communications et décisions de l'Union européenne que la recherche de l'équilibre financier de l'économie, et le bon développement d'un marché des capitaux unique dans l'Union, participe de l'intérêt général européen. Enfin, sur le plan national, il est admis que l'Autorité des marchés financiers assume une mission " d'intérêt général " dès lors qu'elle est chargée d'assurer la protection et le bon fonctionnement des marchés financiers, qu'elle dispose d'un pouvoir de sanction et que son règlement général fait l'objet d'un arrêté d'homologation du ministère concerné. Faute d'une notion centrale de l'intérêt général, il se pourrait finalement que celui-ci puisse être identifié sans passer par la délibération politique mais par l'entreprise transnationale.

\section{LA NORMALISATION COMPTABLE INTERNATIONALE NO SOCIALE DES ENTREPRISES}

La dilution du pouvoir de régulation publique dans l'économie mondiale, ainsi que la domination de la sphère économique et son extension à la sphère juridique et politique, a fait émerger l'entreprise transnationale comme entité politique. Parce les entreprises transnationales agissent au niveau planétaire avec une puissance financière parfois proche de celle des États, la mondialisation les a, non seulement émancipées de leur tutelle

32. Règlement (UE) $n^{\circ} 258 / 2014$ préc, $\$ 16$ et $\$ 17$.

33. Préambule $\$ 9$ et article 3 (B).

34. P. Hérault, Penser l'intérét européen, mémoire Master II Sciences Po, 2009, p.102. 
mais elle les a hissées au niveau même des États dont elles organisent la mise en concurrence, en privilégiant, dans leur gestion, ceux offrant les normes les moins contraignantes. Dès lors, l'idée s'impose progressivement que l'entreprise est en réalité une entité politique originale et, parce qu'elle est ignorée du droit qui ne l'appréhende quà travers le contrat de société et la personnalité morale, elle doit être, en tant que telle soumise à des principes constitutionnels de type nouveau. Le développement de la Responsabilité sociale des entreprises (RSE) participe de ce mouvement. Définie par la Commission européenne comme "la responsabilité des entreprises vis-à-vis des effets qu'elles exercent sur la société ${ }^{35}$ ", la RSE vise à la prise en compte, par les entreprises, des préoccupations sociales et environnementales dans leurs activités et leur stratégie. Ainsi les entreprises sont invitées à optimiser la création d'une communauté de valeurs pour leurs actionnaires, mais aussi pour les autres parties prenantes et l'ensemble de la société. Ce aisant, la responsabilité sociale des entreprises propose de réconcilier les bjectifs économiques et financiers de l'entreprise avec ceux de la société et, pécialement, des parties prenantes à travers la publication, par les entrerrises, d'informations sociales et environnementales à côté des publications l'informations financières. Parce qu'elle propose d'articuler performance ociale et performance financière, la RSE porte ainsi une critique interne de $\neq$ vision actionnariale de l'entreprise en défendant un mode plus partenarial e gouvernance. De façon plus globale, elle oblige à repenser les finalités de entreprise en rapport avec l'intérêt général.

Mais c'est ici révéler un nouveau défi, tant le risque est grand de voir merger de nouveau dans le jeu de la régulation, une concurrence entre s acteurs avec un nouvel éclatement de l'intérêt général et les mêmes iterrogations sur la légitimité du processus. Ainsi parallèlement à une ttervention législative, en France, portée par l'intérêt général et imposant, Ix plus grandes sociétés la publication d'informations non financières, parallèlement à une directive européenne du 22 octobre $2014^{36}$ relare à la publication d'informations non financières et porté par l'intét public européen, des acteurs privés émergent comme normalisateurs proposant des principes pour la diffusion d'informations non finantres. Le plus connu est sans doute la Global Reporting Initiative (GRI), d'acteurs privés du milieu comptable. Si son objectif est de rendre « le

35. Communication du 25 octobre 2011, " Responsabilité sociale des entreprises, une nouvelle régie de l'UE pour la période 2011-2014".

36. 2014/95/UE.
La normalisation comprable internationale: de l'Éat de droit an droit san l'État Yoonne Muller-Lagarde 
reporting de développement durable comparable au rapport financier ${ }^{37}$ ", il s'adresse clairement à l'ensemble des parties prenantes et vise à satisfaire leurs besoins.

Or, un autre référentiel, l'Integrated Reporting (IR), également porté par des acteurs privés, reconnu dans le monde de la comptabilité et de la finance, est né en 2013. S'inscrivant dans le mouvement Business case développé par le World Business Council for Sustainable Development qui rassemble 190 multinationales, il propose une vision instrumentale de la RSE perçue comme un moyen stratégique de création de la richesse intégrant dans le rapport d'activité financier les informations environnementales et sociales. La "maximisation de la valeur éclairée " fournirait notamment à l'entreprise un avantage compétitif sur le marché des ressources et des produits et permettrait d'asseoir sa réputation. Les destinataires restent ici avant tout les investisseurs et apporteurs de capital financier. Or, le 7 février 2013, l'IASB et l'International Integrated Reporting Council (IIRC) ont annoncé la conclusion d'un accord pour renforcer leur coopération sur les travaux de l'IIRC afin d'élaborer un cadre de reporting intégré de l'entreprise. Dans ce contexte encore neuf, la question émerge de savoir si le reporting intégré traduit la réelle volonté d'une économie plus durable ou s'il n'est que la récupération de la RSE par les tenants d'une vision financière de l'économie.

On le devine, la rencontre de la normalisation comptable internationale et de la RSE ouvre un nouveau champ de réflexion.

37. M. Capron et $\mathrm{E}$. Quairel-Lanoizelée, L'Entreprise dans la société, Paris, La Découverre, 2015, p. 197. 